\title{
Forschungs- und technologiepolitische Strategien und Schwerpunkte zu Energie ${ }^{1}$
}

\author{
F. Fahringer
}

Mit der "Überwindung" der Ölkrisen in den 1970er und frühen 1980er Jahren geriet die Sorge um begrenzte Ressourcen in Vergessenheit. Erst der drastische Ölpreisanstieg bis zu einem Preis von 147 Dollar pro Barrel und die Versorgungskrisen infolge von Gaskonflikten haben die Bedeutung des Themas sichere und langfristige Energieversorgung wieder in den Mittelpunkt des Interesses gerückt. Besonders der deutliche Warnruf der Internationalen Energieagentur (IEA) in ihrem World Energy Outlook 2008 bildet einen Anlass zu einer Neubewertung der mittel- bis langfristigen Versorgungssicherheit. Erstmals rechnet die IEA nicht mehr mit einem langfristigen Rückgang der Ölpreise, sondern im Gegenteil mit einem Anstieg, im Zeitraum 2008 bis 2020 im Mittel auf 100 Dollar pro Barrel.

Der europäische SET-Plan (Strategischer Energie-Technologie-Plan für Europa) stellt sich dieser Herausforderung und versucht, bis 2020 die Problematik der Energieversorgung Europas durch die Einführung neuer Energietechnologien zu lösen. Die Umsetzung des SET-Plans erfolgt durch insgesamt sieben europäische Industrie-
Initiativen, die in der Form öffentlich-privater Partnerschaften (public private partnerships) die Demonstration und Markteinführung wichtiger Energietechnologien vorantreiben. Es wurden bereits Industrie-Initiativen zu den wichtigsten Technologien zur Nutzung erneuerbarer Energien sowie der Kernenergie gestartet. Seit dem 21. Juni 2011 läuft auch der Aufbau der Industrie-Initiative SMART CITIES, die der Demonstration von Energieeffizienztechnologien gewidmet ist und für die derzeit die Errichtung einer europäischen Stakeholder-Plattform erfolgt. Sie zeichnet sich dadurch aus, dass dabei nicht nur Forschung und Industrie, sondern auch Städte in eine Umsetzungspartnerschaft einbezogen werden.

Die besondere Herausforderung bei diesem Thema ist, dass es sich bei Urban Technologies nicht um eine Einzeltechnologie handelt, sondern um die Integration unterschiedlicher technologischer Ansätze in ein hocheffizientes Gesamtsystem. Nur so können umfassende Energieeffizienz und verbraucherseitige Maßnahmen im größeren Umfang umgesetzt werden. Unterschiedliche Akteure müssen in solchen Projekten eng zusammenarbeiten.
Kurzfassung eines Vortrags der 49. Fachtagung der Österreichischen Gesellschaft für Energietechnik (OGE) im OVE, die am 20. und 21. Oktober 2011 in Innsbruck stattfindet.

Fahringer, Fritz, Bundesministerium für Verkehr, Innovation und Technologie, Abteilung für Energie- und Umwelttechnologien, Renngasse 5, 1010 Wien, Österreich (E-Mail: fritz.fahringer@bmvit.gv.at) 